\title{
Two families with autosomal recessive spastic paraplegia, pigmented maculopathy, and dementia
}

\author{
Stewart Webb, Victor Patterson, Michael Hutchinson
}

\begin{abstract}
Objective-Two families with autosomal recessive hereditary spastic paraplegia and pigmented maculopathy are described.

Methods-All family members were examined by two neurologists. An assessment of cognitive function in affected members was made using the mini mental state examination (MMSE) or Cambridge cognitive examination (CAMCOG).

Results-Six patients from two families presented with a slowly progressive, autosomal recessive, spastic tetraplegia. Although they were always considered to be intellectually slower than their peers, further intellectual deterioration was noted during the second decade. Five had a pigmented maculopathy with mild decrease in visual acuity and all had distal amyotrophy, mild cerebellar signs, and developed faecal and urinary incontinence late in the course of the disease.

Conclusion-The association of hereditary spastic paraplegia and pigmented maculopathy has rarely been described; only 11 families with 32 affected members have been reported, showing considerable heterogeneity in presentation. These described conditions may be allelic or more probably reflect mutations at different genetic loci.
\end{abstract}

(F Neurol Neurosurg Psychiatry 1997;63:628-632)

Keywords: hereditary spastic paraplegia; maculopathy; dementia; Kjellin's syndrome

Department of Neurology, St Vincent's Hospital, Elm Park, Dublin 4, Ireland $S$ Webb

$M$ Hutchinson

Department of Neurology, Royal Victoria Hospital, Belfast, BT12 6BA, Northern Ireland

V Patterson

Correspondence to: Dr Stewart Webb, Department of Neurology, St Vincent's Hospital, Elm Park, Dublin 4, Ireland.

Received 21 October 1996 and in final revised form 30 April 1997

Accepted 6 May 1997
Since the original description of hereditary spastic paraplegia, reports of complicated forms have been limited only by the finite way in which neurological disease can present. ${ }^{1}$ Until the underlying genetic and biochemical defects are established, classification must rely on accurate clinical description. The association of hereditary spastic paraplegia and pigmentation of the macula has rarely been described. Associated clinical features may include hearing loss, mental retardation, dementia, behavioural disturbance, cerebellar signs, distal amyotrophy, ichthyosis, and syndactyly.

Kjellin described two families with autosomal recessive inheritance, mental retardation, and onset of spastic paraplegia in the third decade, associated with macular pigmentation and distal amyotrophy. ${ }^{2}$ In this study two families are described with clinical features of Kjel- lin's syndrome but with several distinguishing points: the age of onset for spastic paraplegia was earlier and they had additional clinical features including sphincter disturbances, dysarthria, mild cerebellar ataxia of the upper limbs, and further intellectual deterioration in the second decade that were not described by Kjellin.

\section{Methods}

Members from both families were examined by two neurologists. An assessment of cognitive function in affected members was made using the mini mental state examination (MMSE) or Cambridge cognitive examination (CAMCOG). ${ }^{3}$ Scores in the CAMCOG are rated out of 107 ; $>80$ being considered normal, 60-79 mild dementia, 35-59 moderate dementia, and $<35$ severe dementia.

Affected family members had the following tests performed: a full blood count, blood film and differential, erythrocyte sedimentation rate and karyotyping, serum levels of urea and electrolytes, liver function tests, glucose, creatine phosphokinase, aldolase, thyroid function tests, vitamin B12 and folate, vitamin E, vitamin A, cholesterol and triglycerides, fasting pyruvate and lactate, copper, caeruloplasmin, lead, acanthocytes, immunoglobulins, syphilis serology, urinalysis for reducing sugars, protein, phenylketouria, acetest, amino acid chromatography, calcium, mucopolysaccharides, urobilinogen, porphyrins, indican, a chest radiograph and ECG, echocardiography (ECHO), analysis of CSF including oligoclonal bands, EEG, visual evoked responses (VER), electroretinography(ERG), fundalphotography, nerve conduction studies, EMG, and neuroimaging, including MRI of the brain and spinal cord.

THE FAMILIES

Both families (fig 1A and B) lived within $35 \mathrm{~km}$ of each other. In family $A$, there were three affected (IV-2A, IV-5A, IV-6A) and four unaffected children (IV-1A, IV-3A, IV-4A, IV-7A). Their sister IV-1A was normal until she developed Rassmussen's encephalitis at 30 years of age. Their mother, III-12A, was normal and their father, III-11A who died aged 55, was reported to have been normal. Consanguinity was denied and there was no other affected member on either side of the family.

In Family $B$ there were three affected (IV-1B, IV-2B, IV-3B) and three unaffected children (IV-4B, IV-5B, IV-6B). Their sister IV-4B had intellectual impairment after an episode of meningitis aged 4 months but was 
otherwise normal. Their parents were first cousins and were normal on examination. There was no history of similar clinical features on either side of the family.

CASE REPORTS

The affected members within each family had similar phenotype and clinical course (table 1). The following case reports are representative of each family.

Family $A$

Case $I V-2 A$ - This 39 year old man was born by forceps delivery. He was considered a "slow learner" and a psychologist reported borderline mental retardation when he was eight years old. His intellect showed further deterioration at 15 years and he developed a slowly progressive spastic paraplegia at 17 years. For the past year he has been confined to a wheelchair with both urinary and faecal incontinence. At 34 years he developed hallucinations, paranoid ideation, and mood swings. His present medications include thioridazine, benztropine, monthly injections of flupenthixol, and baclofen.

On examination he had pronounced intellectual impairment and his speech was dysarthric, impoverished, and lacked spontaneity. Lateral eye movements were slightly reduced. At 39
II

I

III

IV

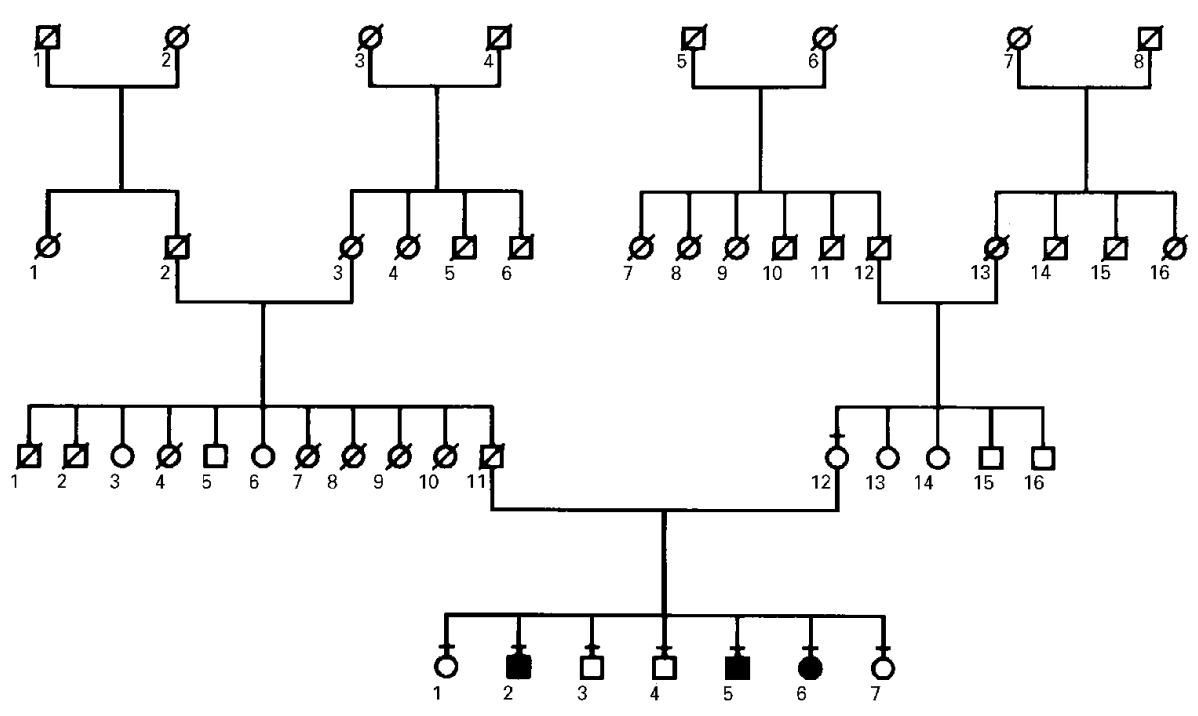

Family B

II

III

IV

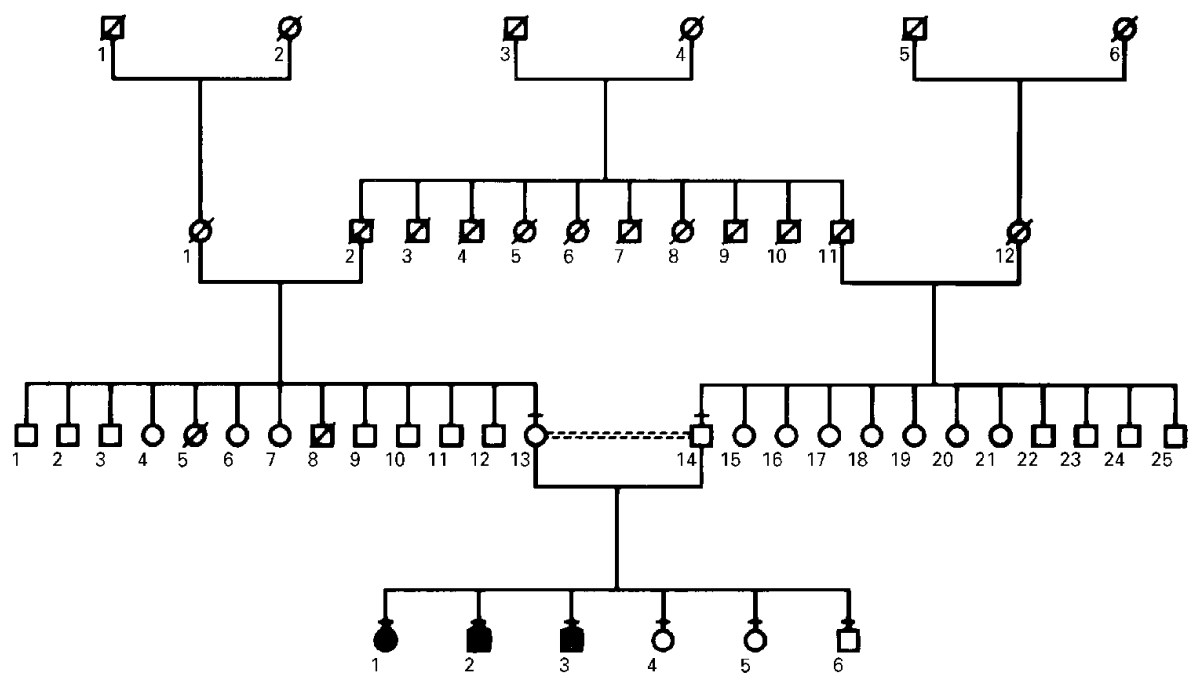

Family $A$

Figure l (A) (Family A) and (B) (Family B). Hereditary spastic paraplegia and pigmented maculopathy. Filled symbols indicate affected members. Horizontal bars above symbols indicate fully examined members of the family. 
Table 1 Clinical details of families $A$ and $B$

\begin{tabular}{lllll}
\hline Case & Age & HSP onset & Clinical features & Other features \\
\hline IV-2A & 39 & 17 & All members have macular & Psychosis \\
IV-5A & 33 & 18 & pigmentation (except IV-3B), & Psychosis \\
IV-6A & 32 & 23 & mental retardation, dementia, & Nil \\
IV-1B & 33 & 13 & distal amyotrophy, mild & Epilepsy \\
IV-2B & 32 & 14 & cerebellar signs, and bladder & Nil \\
IV-3B & 30 & 16 & and bowel incontinence & Dystonia \\
\hline
\end{tabular}

HSP $=$ Hereditary spastic paraplegia. years and was confined to a wheelchair at 21 years. In the past two years he has developed faecal and urinary incontinence. He was always considered by his parents to be intellectually slower than the other children and had further cognitive deterioration in the second decade.

On examination he sat slumped forward in his wheelchair, being unable to support himself in a sitting position. $\mathrm{He}$ had pronounced kyphosis, rounded shoulders, and had difficulty keeping his head up. There was no spontaneous speech and responses were slow, monosyllabic, and dysarthric with a nasal quality. His mother thought that his vision was normal but formal assessment was not possible. At 24 years ophthalmological examination disclosed clumps of macular pigmentation bilaterally, each surrounded by areas of atrophic change. His arms showed fasciculations with considerable wasting of the thenar and to a lesser extent the hypothenar eminence. He had mild contracture deformity at both elbows, tone was mildly increased on the left side, and reflexes were brisk bilaterally. He had reduced power and lack of spontaneous movement in both arms. Although he reached out for objects in front and to the side of him without any tremor he was unable to lift his arms above his head. There was wasting with fasciculations in his calf muscles, tone was cosiderably increased with adductor spasm, and power was absent. Both achilles tendons were shortened. Reflexes were brisk at the knees but absent at the ankles. His planter responses were extensor and his feet were flat.

\section{Results}

FAMILY A

The CAMCOG score was 35 in IV-2A, 46 in IV-5A, and 56 in IV-6A. Brain MRI showed

Case IV-2B-This 32 year old man developed a slowly progressive spastic paraplegia at 14

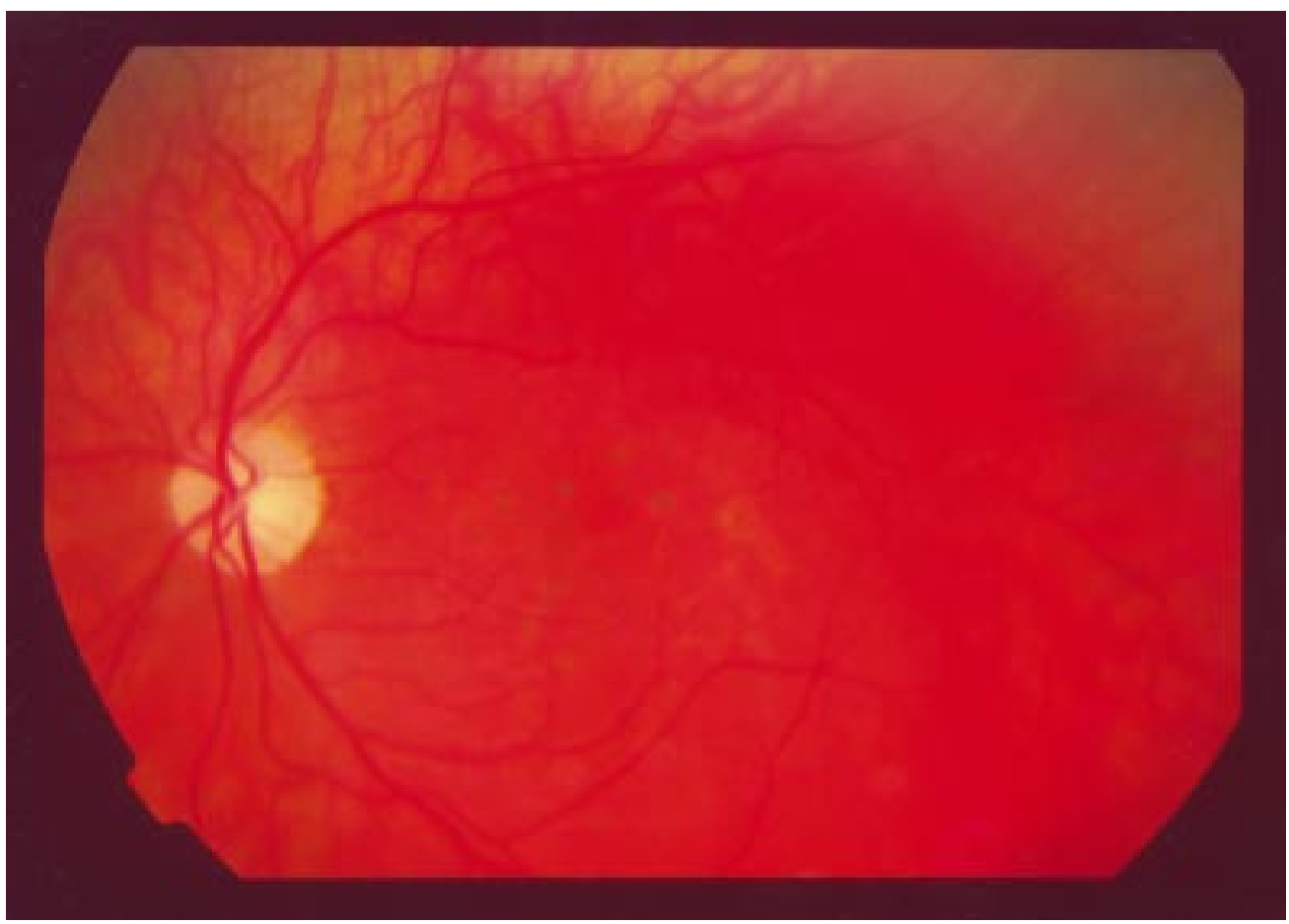

Figure 2 Fundoscopy in case IV-6A shows several discrete areas of pigment aggregation in the macula each surrounded by a yellow atrophic halo. 
diffuse atrophy of the cerebral hemispheres, corpus callosum, and brain stem in all three affected members. The EEG was normal in IV-2A but showed non-specific voltage changes and slow wave activity in IV-5A and IV-6A. Measurements in CSF including oligoclonal banding was normal in IV-6A. The VER was normal in IV-5A but showed increased latency in IV-2A and IV-5A. The ERG was normal in IV-2A but the rod isolated responses were delayed and the amplitude was reduced in IV-5A and IV-6A and the cone responses were normal but the amplitude was reduced in IV-6A. Nerve conduction studies were normal but the EMG showed fasciculations in the small muscles of the hand in IV-6A. The ECG was normal in IV-5A but there was right axis deviation with incomplete right bundle branch block in IV-2A and generalised T wave flattening in IV-6A. An ECHO was normal in IV-6A. A muscle biopsy, in IV-6A, showed random fibre atrophy and fibre type grouping indicative of denervation and associated re-innervation. There were no histological features to support a mitochondrial disorder. All other tests were normal.

FAMILY B

The MMSE score was $17 / 38$ in IV-3B, but IV-1B and IV-2B were unable to perform this test. Brain CT showed diffuse cerebral atrophy in IV-1B and IV-3B. The EEG was normal in IV-2B but showed generalised slow wave activity in IV1B. Measurements in CSF was normal in IV-1B. The VER showed increased latency in both eyes but pattern ERG was normal in IV-3B. All other tests were normal.

\section{Discussion}

Six members from two families have an autosomal recessive syndrome characterised by mental retardation, further intellectual deterioration in the second decade, spastic tetrapaparesis, distal amyotrophy, macular pigmentation, mild reduction in visual acuity, dysarthria, mild cerebellar signs, and faecal and urinary incontinence (table 1).

These two families, although unknown to each other, are probably related, as they have an identical, rare, autosomal recessive clinical syndrome and have lived within $35 \mathrm{~km}$ of each other for over five generations.

All affected members were considered by their parents to be intellectually slow as children and to have further cognitive deterioration in the second decade. The earlier the age of onset and the longer the duration of spastic tetraparesis the more severe was the cognitive impairment.

They all developed an abnormal gait between 13 and 23 years of age and progressed to using a wheelchair within three to 21 years. All had a similar pattern of muscle weakness in the upper body, being unable to support themselves in a sitting position and having reduced power for neck flexion, elbow extension, and finger and thumb abduction. All affected members had evidence of a lower motor neuron lesion in the arms, probably of anterior horn cell origin.

Macular pigmentation was seen in five affected members and although lesions predominantly involved the macula, in two members more widespread retinal pigmentation was seen. In the four cases presented by Kjellin extension of pigmentation outside the macula was not seen at fundoscopy but in all cases an

Table 2 Autosomal recessive hereditary spastic paraparesis (HSP) and macular pigmentation - summary of previous reports

\begin{tabular}{|c|c|c|c|c|c|c|c|c|}
\hline Author (ref) & Pedigree & $\begin{array}{l}\text { HSP onset } \\
(y)\end{array}$ & $\begin{array}{l}\text { Mental } \\
\text { retardation }\end{array}$ & Dementia & Fundal lesions & $\begin{array}{l}\text { Amyotrophy } \\
\text { of arms }\end{array}$ & $\begin{array}{l}\text { Cerebellar } \\
\text { signs }\end{array}$ & Other features \\
\hline \multirow[t]{6}{*}{ Webb et al, present study } & 1 & 17 & Yes & Yes & Yes & Yes & Yes (mild) & Psychosis \\
\hline & & 18 & Yes & Yes & Yes & Yes & Yes (mild) & Psychosis \\
\hline & & 23 & Yes & Yes & Yes & Yes & Yes (mild) & Nil \\
\hline & 2 & 13 & Yes & Yes & Yes & Yes & Yes (mild) & Epilepsy \\
\hline & & 14 & Yes & Yes & Yes & Yes & Yes (mild) & Nil \\
\hline & & 16 & Yes & Yes & No & Yes & Yes (mild) & Focal dystonia \\
\hline \multirow[t]{2}{*}{ Farmer et al ${ }^{14}$} & 1 & 13 & No & Yes & Yes & Yes & No & Dysarthria \\
\hline & & Teens & No & Yes & Not known & Yes & No & No \\
\hline \multirow[t]{3}{*}{ Sjaastad et al $\bar{l}$} & 1 & 5 & No & Yes & Yes & No & Yes (mild) & No \\
\hline & & 29 & No & Yes & Yes & No & Yes (mild) & No \\
\hline & & 28 & No & Yes & Yes & No & No & No \\
\hline \multirow[t]{5}{*}{ Mahloudji and Chuke ${ }^{6}$ and Farmer et $a l^{4}$} & 1 & 35 & Yes & No & Yes & No & No & No \\
\hline & & 30 & Yes & No & Yes & No & Yes (mild) & No \\
\hline & & 35 & No & No & No & No & No & No \\
\hline & & 30 's & No & Yes & Yes & Yes (mild) & No & Dysarthria \\
\hline & & 30 & No & Yes & Yes & Yes (mild) & No & Dysarthria \\
\hline \multirow[t]{4}{*}{ Ledic and Van Bogaert ${ }^{7}$} & 1 & 10 & No & Yes & Yes & Yes & No & Hearing loss \\
\hline & & 12 & No & No & No & No & No & Sensation loss \\
\hline & & 12 & No & No & Yes & Yes & No & No \\
\hline & & Not known & No & No & Yes & No & No & No \\
\hline \multirow[t]{4}{*}{ Kjellin $^{2}$} & 1 & 30 & Yes & No & Yes & Yes & No & Hearing loss \\
\hline & & 23 & Yes & No & Yes & Yes & No & Hearing loss \\
\hline & 2 & 23 & Yes & No & Yes & Yes & No & No \\
\hline & & 24 & Yes & No & Yes & Yes & No & No \\
\hline \multirow[t]{3}{*}{ Sjogren and Larsson ${ }^{8}$} & 1 & Infancy & Yes & No & Yes & No & No & Ichthyosis \\
\hline & & Infancy & Yes & No & Yes & No & No & Ichthyosis \\
\hline & 2 & Birth & Yes & No & Yes & No & Not known & Ichthyosis \\
\hline \multirow[t]{3}{*}{ Louis-Bar and Pirot ${ }^{9}$} & 1 & 12 & No & No & Yes & Yes & No & Syndactyly \\
\hline & & $<10$ & Not known & Not known & Yes & Not known & Not known & Syndactyly \\
\hline & & Not known & No & Mo & No & No & No & Syndactyly \\
\hline \multirow{2}{*}{ Schaffer ${ }^{1011}$} & 1 & 3 & No & No & Yes & No & Yes & No \\
\hline & & 3 & No & No & No & No & Yes & No \\
\hline
\end{tabular}


ERG indicated more widespread retinal involvement. ${ }^{2}$ The maculopathy may be present at the onset of the spastic paraparesis. More commonly, however, it develops several years after the spastic paraparesis has begun. For example, in IV-5A spastic paraparesis began at 18 years of age; initial fundoscopy at 25 was normal but repeat examination at 33 showed a macular pigmentation. These macular lesions progressed slowly-for example, in IV-6A visual acuity was unchanged and only a few additional macular lesions were found after an eight year interval. The late onset and slow progression of macular lesions were also found by Kjellin. ${ }^{2}$ The visual acuity was mildly affected in all our cases and either slowly deteriorated or remained static.

Additional features were present in some affected members, tonic-clonic seizures in IV-1B, dystonic movements of the head and hand in IV-3B, and hallucinations, paranoid ideation, and mood swings in both IV-2A and IV-5A.

The association of hereditary spastic paraplegia and pigmented maculopathy has rarely been described, yet there is considerable clinical heterogeneity among the few reported cases (table 2). The two families reported in this study are similiar on clinical grounds but differ from all previously reported cases, including the two families described by Kjellin. This phenotypic variation may reflect different mutations at a single gene locus or mutations at several different loci. Molecular genetic studies are required to provide a definite answer. Although each report is different many similarities can be seen. All reported families (except one sporadic case) have autosomal recessive inheritance. The onset of spastic paraplegia may range from three to 35 years but within each family the onset is tightly clustered (table 2). The presence or absence of specific clinical features varies between families but is relatively constant within each family. Although the macula is predominantly affected by disease, there is often evidence of more widespread retinal involvement both clinically and on ERG. The maculopathy usually occurs late and is slowly progressive or static. Except for the reports by Ledic and Van Bogaert ${ }^{7}$ and by Schaffer, ${ }^{10}{ }^{11}$ the effect on visual acuity is usually mild. Amyotrophic changes are common and affect distal musculature, more in the arms then in the legs and particularly the thenar eminence. Cerebellar disturbance is mild or absent. Dementia has been described before in some of these families but has not been recognised as a feature of Kjellin's syndrome. Spastic paraparesis, pigmented maculopathy, and dementia may occur together in other diseases (for example, Hallervorden-Spatz disease and LawerenceMoon-Biedl disease). Although these conditions should be included in any differential diagnosis, they are characterised by predominant clinical and pathological features other then spastic paraparesis and have therefore been excluded from table 2. HallervordenSpatz disease is an autosomal recessive condi- tion in which spastic paraparesis, pigmented maculopathy, mental retardation, and dementia may occur together. The usual presentation and dominant clinical features are an akineticrigid and dystonic syndrome beginning between 7 and 12 years of age. Brain MRI may also show the presence of iron in the globus pallidus and substantia nigra. LawerenceMoon-Biedl disease is an autosomal recessive condition characterised by retinitis pigmentosa, mental retardation, obesity, hypogonadism, and polydactyly; spastic paraparesis may be a variable, late finding. Batten's disease is an autosomal recessive condition characterised by a progressive visual loss, pigmented retinopathy, and dementia with periodic acid Schiff positive material in neurons. Spastic paraparesis, and extrapyramidal and cerebellar signs may occur late in this condition. Subacute necrotising encephalomyelopathy (Leigh's disease) is a mitochondrial disorder of pyruvate metabolism and may present with developmental arrest, retinal pigmentation, optic atrophy, loss of vision, dementia, ophthalmoplegia, nystagmus, dystonia, seizures, respiratory abnormalities, and spastic or flaccid paraparesis. Brain MRI may show areas of necrosis in the basal ganglia and putamen.

In summary, two families have been described with autosomal recessive hereditary spastic paraplegia and a pigmented maculopathy. All had mental retardation and developed a progressive spastic paraplegia in the second decade associated with further cognitive deterioration, a pigmented maculopathy, and amyotrophic changes. This condition may be easily missed, unless specifically sought, as only a mild reduction in visual acuity is produced and the pigmented maculopathy may occur several years after the onset of the spastic paraplegia. Therefore, we suggest that all autosomal recessive and sporadic cases of hereditary spastic paraplegia should have an ophthalmological examination performed with dilatation of the pupils.

1 Sutherland JM. Familial spastic paraplegia. In: Vinken PJ, Bruyn GW, eds. Handbook of clinical neurology. Amsterdam: North Holland, 1975;22:421-31.

2 Kjellin K. Familial spastic paraplegia with amyotrophy, oligophrenia and central retinal degeneration. Arch Neurol 1959;1:133-40.

3 Roth M, Tym E, Mountjoy C, et al. CAMDEX. Br $\mathcal{F}$ Psychiatry 1986;149:698-709

4 Farmer SG, Longstreth WT, Kalina RE, Todorov AB. Fleck retina in Kjellin's syndrome. Am f Ophthalmol 1985;99:4550.

5 Sjaastad O, Berstad J, Gjesdahl P, Gjessing L. Homocarnosinosis. 2. A familial metabolic disorder associated with spastic paraplegia, progressive mental deficiency and retinal pigmentation. Acta Neurol Scand 1976;53:275-90.

6 Mahloudji M, Chuke P. Familial spastic paraplegia with retinal degeneration. fohns Hopkins Hospital Bulletins 1968; 123:142-4.

7 Ledic P, Van Bogaert L. Heredodegenerescence cerebelleuse et spastique avec degenerescence maculaire. $f$ Genet Hum 1960;9:140-57.

8 Sjogren T, Larsson T. Oligophrenia in combination with congental ichthyosis and spastic disorders. A clinical and genetic study. Acta Psychiatr Scand 1957;113(suppl1):112.

9 Louis-Bar D, Pirot G. Sur une paraplegie spasmodique avec Louis-Bar D, Pirot G. Sur une paraplegie spasmodique avec
degenerescence maculaire chez deux freres. Ophthalmodegenerescence maculair

10 Schaffer K. Zur Pathologie und pathologischen Histologie der spastischen Heredo-degeneration (hereditare spastische Spinalparalyse) Deutsche Ztschrift Nervenh 1922;73: 101-28.

11 Schaffer K. Zur Histopathologie der idiotypischen lateral Sklerose oder spastischen Heredodegeneration. Arch Psychiatry 1926;77:675-97. 